\section{COVID-19: measures to protect older adults from SARS-CoV-2 infection}

\author{
Israel Oluwasegun Ayenigbara, \\ Olawale Akanbi Moronkola \\ School and Community Health \\ Education Unit, Department of Health \\ Education, University of Ibadan, \\ Ibadan, Nigeria
}

\section{Abstract}

As every nation battles the coronavirus disease 2019 (COVID-19) pandemic comprehensively, older adults are by far the most affected group in terms of morbidity and mortality rates. Particularly individuals in the age range of $>60$ years and with comorbidities and other geriatric conditions are at heightened risk of complications from COVID-19 compared to any other age groups in the world. The time between the infection of severe acute respiratory syndrome-coronavirus 2 (SARSCoV-2) and the manifestation of the symptoms is between two to twelve days. Typical symptoms of COVID-19 are high temperature, dry cough, and breathing difficulties in complicated cases, while new evidence shows atypical presentations of COVID-19 symptoms in older adults, and are highlighted in this review. From this synopsis, we deduced that firstly, the severity of COVID-19 among older adults is because of biological (dwindling immunity with old age), socio-economic (poverty and over-stretched health system) and physical reasons (frailty and comorbidities). Secondly, there is an upsurge in the rates of transmission and COVID-19 fatalities in nursing homes globally. Lastly, to abate the spread of SARS-CoV-2 among older adults, strict adherence to physical distancing, frequent hand hygiene and respiratory hygiene, frequent disinfection of surfaces, stoppage to unnecessary travel and nonessential hospital's visitations, appropriate use of face masks, healthy life style choices, proper identification and isolation of infected older adults, assistance and support for older adults in the community, and prevention of infections in nursing homes should all essentially be implemented globally. It is recommended that maximum compliance to measures provided in this review should be ensured and implemented. Governments, civil societies and general public should provide supports for older adults during this COVID-19 pandemic period, and new researches should look more into the global severity of COVID-19 on nursing homes and the geriatric populations generally to get progressively feasible exact information that will enable informed preventive choices.

\section{Introduction}

Coronavirus disease 2019 (COVID$19)$ is a nascent and virulent plague, which originated in Wuhan, China, in late 2019. COVID-19 is caused by a highly infectious pathogen [severe acute respiratory syndrome-coronavirus 2 (SARS-CoV-2)], with biological descriptions of a positive sense, single-strand virus, and with an encased ribonucleic acid (RNA), with affinity to coronaviridae viruses. ${ }^{1,2}$ In late memory, the SARS-CoV and the Middle East respiratory syndrome (MERS)-CoV are some remarkable coronaviridae viruses that have caused diseases in humans. ${ }^{3}$ Coronaviruses are diseases causing pathogens in bats and other animals, and current evidence firmly proposes that the novel SARS-CoV-2 is a zoonosis of pangolins' and bats' SARS-like coronavirus of the beta-coronavirus family, subgenus Sarbecovirus. ${ }^{4}$

As a worldwide general medical issue, COVID-19 has spread endlessly to the majority of nations on the planet, with contact with respiratory and bodily fluids of patients as the major contagion route. ${ }^{5}$ Although there are odds of airborne spread of SARS-CoV-2 as nosocomial transmission, in any case there is no strong proof to validate this claim. ${ }^{6}$ The onset of COVID-19 symptoms, although not consistent, is normally between two to twelve days, and typical symptoms are high temperatures (in $44 \%$ to $98 \%$ cases), dry coughs (in $46 \%$ to $82 \%$ cases), breathing difficulties (in $31 \%$ of cases), muscle pains and weakness (in $11 \%$ to $44 \%$ cases), and anosmia, while pharyngitis, cerebral pain, expectoration of sputum and wet productive cough, and gastro-intestinal issues are minor signs of COVID-19. ${ }^{6}$ The older population has shown globally more severity in signs and symptoms of COVID-19 compared to any other age group because of obvious reasons. ${ }^{6}$ Because of the high volatility and infectivity of SARS-CoV-2, COVID-19 was declared a pandemic by the World Health Organization..$^{5-7}$ Presently, COVID-19 has been accounted for in many nations, and older adults, particularly those with comorbidities and other geriatric diseases, are internationally highly affected in all
Correspondence: Israel Oluwasegun Ayenigbara, School and Community Health Education Unit, Department of Health Education, University of Ibadan, Ibadan, Nigeria.

Tel.: +2348139177538.

E-mail: histrealite2647@gmail.com

Key words: COVID-19; pandemic; SARSCoV-2; prevention; older adults; nursing homes.

Acknowledgements: We appreciate the authors of the articles reviewed for impacting on our ideas and unknown reviewers of the draft of the paper, as all enriched the final outcome of the published version of this article

Conflict of interests: the authors declare no potential conflict of interests.

Availability of data and materials: not required

Ethics approval and consent to participate: not required.

Dedication: The article is dedicated to all brave front-line workers who are ensuring the safety, protection, care and treatment of older adults as well as individuals in the risk of COVID-19 contagion.

Received for publication: 17 April 2020.

Revision received: 25 June 2020.

Accepted for publication: 25 August 2020.

This work is licensed under a Creative Commons Attribution-NonCommercial 4.0 International License (CC BY-NC 4.0).

${ }^{\circ}$ Copyright: the Author(s), 2020

Licensee PAGEPress, Italy

Geriatric Care 2020; 6:9045

doi:10.4081/gc.2020.9045

cases scenarios. ${ }^{5,6,8}$ For example, in the European mainland, over $95 \%$ of all COVID-19 fatalities have occurred in individuals of $>60$ years, while older individuals of $>80$ years represent over half of the fatalities recorded ${ }^{8}$ Besides, eight out of ten COVID-19 fatalities occurs in older patients because of comorbidities (generally heart diseases/hypertension and diabetes). ${ }^{8}$ The aforementioned conditions are evident in Nigeria and in most nations as individuals who have COVID-19 or died from it were older adults with existing chronic sicknesses and other geriatric diseases. The previously mentioned insights are alarming and stunning, thus, the critical requirement for the review on viable preventive measures to shield older individuals from SARS-CoV-2 infection. 


\section{Sources of information}

The updated literature review was gotten from PubMed databases, and various World Health Organization and Center for Diseases Control and Prevention archives on the title of discourse were counseled. Furthermore, recommendations and opinions were gotten from experts in the field of Heath Education and promotion, and infectious diseases specialists.

\section{COVID-19 pandemic and older adults}

Various researches have certified that individuals in the age range of $>60$ years and with comorbidities and other geriatric conditions, for instance cardiovascular disease, diabetes, malignancy, lung disease, Alzheimer disease, dementia, and delirium, are at heightened danger of complications from COVID-19 and SARS-CoV-2 infection compared to any other age groups in the world. ${ }^{8-12}$ A comprehensive model based on the confirmed cases of SARS-CoV-2 infection and fatalities from COVID-19 uncovered that globally, COVID-19 fatality rate is over $13.4 \%$ in patients of $>80$ years old, compared to $8.6 \%, 4 \%, 1.25 \%$ and $0.3 \%$, of patients in their $70 \mathrm{~s}, 60 \mathrm{~s}, 50 \mathrm{~s}$ and $40 \mathrm{~s}$ respectively, this confirms the lethality of COVID-19 in older adults. ${ }^{13}$ Over $59 \%$ of adults within the age group of 75 to 84 years with confirmed cases of COVID-19 in the United States of America (USA) presented serious clinical symptoms that required hospitalization compared to around $21 \%$ of diagnosed patients within the age range of 20 to 44 years. ${ }^{14}$

The severity of COVID-19 in older adults is due to biological, socio-economic and physical reasons. Firstly, older adults ordinarily have more fragile immune system, which makes them more susceptible to contagious infections and diseases. ${ }^{15}$ The white platelets (Leukocytes), antibodies and $\mathrm{T}$ cells are defensive properties that help the body ward off infections and microorganisms. Sadly, because of advance in age, there are continuous decreases in $\mathrm{T}$ cells that the body produces. ${ }^{16}$ For example, during the formative phases of life, the thymus produces more $\mathrm{T}$ cells compared to the numbers created at adolescence; be that as it may, there are substantial decreases in the numbers delivered as one ages, and the lower quantity of $\mathrm{T}$ cells correlates with lower capacities to guard against a partic- ular infection, thus making an individual helpless when the system is attacked by a novel disease, like the SARS-CoV-2. ${ }^{17}$ Furthermore, advance in age lessens the rate and immediacy with which antibodies ( $\mathrm{T}$ cells and $\mathrm{B}$ cells) react to battling diseases in the body. This happens because of huge cytokines storm in older adults. ${ }^{18}$ Fusillade of cytokines attacks the lungs and results in assortment of manifestations, with the most obvious being the acute respiratory distress syndrome (ARDS), a typical cause for COVID-19 fatalities. ${ }^{19-21}$ Likewise, sex is a significant determinates of monstrous cytokines storm in the body, as older men delivers more cytokine-creating cells compared to older women, thus making women to have numerous sound antibodies (B cells and $\mathrm{T}$ cells) to ward off infections than their male counterparts. This correlates with the sex based contrasts in the pandemic, with older men recording more infection cases and COVID-19 fatalities compared to older women. ${ }^{17,22}$ Diminished antibodies (B and $\mathrm{T}$ cells) make the body more fragile, consequently, the reoccurrence of SARS-CoV-2 infection ought to be forestalled as older adults who survived COVID-19 might not have strong immunity should they be exposed to SARSCoV-2 again. ${ }^{17}$

Also, with some COVID-19 preventive measures such as partial lockdown, social distancing and isolation still in place in certain nations of the world, some older adults might likely be in a condition of need or penniless, which may bring about shortage of necessities to care for themselves, for example, shortage of foods and medications to treat and oversee different comorbidities and geriatric conditions. ${ }^{23}$ Numerous older adults are out of employment in numerous nations for evident reasons and, moreover, in many developing nations the provisions of social help is defective, making older adults defenseless against condition of destitution and vulnerability. Presently in the world, numerous older adults are disadvantaged during the pandemic, due to their overwhelmed and compromised health system, their frailty, which if not well managed could cause segregation, which may forestall them the availability to essential COVID-19 preventive information, getting foods and fundamental medication supplies consistently, particularly if they live alone. This is in addition to the fact that some older adults usually have comorbidities and other geriatric conditions, for example, cardiovascular diseases, lung diseases, diabetes, different neurological diseases, kidney diseases, etc., which debilitates the body's capacity to battle diseases, making older adults more vulnerable. ${ }^{24}$

\section{Comorbidities and COVID-19 fatalities among older adults}

Comorbidities could also be described as underlying medical conditions or preexisting health conditions, which implies the occurrence of one or more disease conditions co-occurring, or concomitant to a primary or prior health condition. As COVID-19 spreads internationally, older adults, particularly those with comorbidities and other geriatric conditions, are seriously affected in terms of morbidity and mortality rates. ${ }^{24-29}$ In the USA for example, over $90 \%$ of patients admitted into hospitals are either comorbid or multimorbid. ${ }^{30}$ Furthermore, COVID-19 patients of $>65$ years of age needs hospitalization at the pace of 13.8 per 100,000 , compared to other age groups of 50 to 64 years $(7.4$ per 100,000$)$ and 18 to 49 years $(2.5$ per 100,000$) .{ }^{30}$ Likewise, over $95 \%$ of COVID-19 hospitalized older patients of $>65$ years have comorbidities, compared to patients in the age group of 5064 years $(86.4 \%)$ and $18-44$ years $(85.4 \%) .{ }^{30}$ The most recorded comorbidities from COVID-19 older patients are hypertension $(72.6 \%)$, followed by cardiovascular diseases (50.8\%) and obesity (41\%). ${ }^{30}$ Statistics from New York State uncovered that over $86 \%$ of confirmed COVID- 19 fatalities involved at least one comorbidities, with hypertension the most frequent, followed by diabetes (37.3\%), hyperlipidemia (18.5\%), coronary artery disease $(12.4 \%)$, renal disease $(11 \%)$, dementia (9.1\%), chronic obstructive pulmonary disease (COPD) (8.3\%), malignancy (8.1\%), atrial fibrillation $(7.1 \%)$, and myocardial infarction (7.1\%), as over $63 \%$ of all fatalities occurred in patients of $>70$ years. ${ }^{31}$ Generally, the recovery rates in COVID-19 patients are high, however, recuperation rates are lower in older patients with comorbidities and other geriatric conditions. There have been over nine million affirmed cases of COVID-19 globally and over four hundred thousand fatalities recorded as at 25th June, $2020 .{ }^{32}$ Incredibly, over $90 \%$ of COVID-19 fatalities in the European continent occurred in the most noticeably terrible hit nations of Italy, Spain, France, and the United Kingdom. ${ }^{8,32}$ The general findings from this section underscore the significance of preventive measures to mitigate the ceaseless spread of SARS-CoV-2 among older adults and people with comorbidities and other geriatric conditions. 


\section{Prevention of COVID-19 among older adults}

Reports and insights around the globe unequivocally demonstrate that older adults are far the most affected in the severity of COVID-19 pandemic. This is significant perceptions because the health of older adults is of utmost priority in the world. ${ }^{8,33}$ As the search for a vaccine and standard cure for COVID-19 continues, preventive measures, until further notice, are the main less expensive mitigative measures to repress the spread of SARS-CoV-2 among older adults. ${ }^{6,34}$ The following are effective preventive measures to shield older adults from SARS-CoV-2 infection (Figure 1).

\section{Criteria for prompt testing of older adults}

Quick testing is imperative for the early diagnosis of COVID-19 among older adults to subside deterioration and conditions from exacerbating, and to avert the spread of SARS-CoV-2 to other individuals and community transmission. Typical symptoms of COVID-19, for example fever, cough, and dyspnea, may be missing in older adults regardless of respiratory disease, as just 20$30 \%$ of older patients with infection present with fever. ${ }^{35}$ Current evidence recommends that atypical symptoms, for examples falls, delirium, malaise, generalized weakness, functional decline, anorexia, conjunctivitis, headache, dizziness, increased sputum production, chest pain, hemoptysis, diarrhea, rhinorrhea, nausea, abdominal pain, vomiting, nasal congestion, tachypnea, anosmia, unexplained tachycardia, or hypotension, may be the clinical presentation of COVID19 in older adults. ${ }^{35,36}$ Importantly, the threshold for diagnosing fever ought to be lower (for example $37.5^{\circ} \mathrm{C}$ ) or an expansion of $>1.5^{\circ} \mathrm{C}$ from regular temperature range for older adults. ${ }^{36}$ Understandably, atypical symptoms of COVID-19 in older adults might depend from a few variables, including physiological changes with age, comorbidities, and inability to give a precise history, because older age, frailty, and increasing number of comorbidities increase the likelihood of atypical symptoms. Furthermore, older adults with disabilities and other geriatric conditions are three times at a higher risk of infection or severe illness from COVID-19 than older adults without disabilities on account of their basic ailments. ${ }^{37}$ For example, older adults who have limited mobility, attention difficulties and dyslexia should be engaged in routine COVID-19 testing. ${ }^{37}$

\section{Identification and isolation of infected older adults}

As discussed above, older adults with atypical symptoms ought to be promptly identified and prioritized for COVID-19 testing in the community and nursing homes, as patients may slip past the normal screening process and may inadvertently be admitted to general clinical ward as opposed to a specified COVID-19 section. In the event that diagnosis turns positive, older patients ought to be instantly isolated and treated adequately to prevent the spread of SARS-CoV-2 and exacerbating of

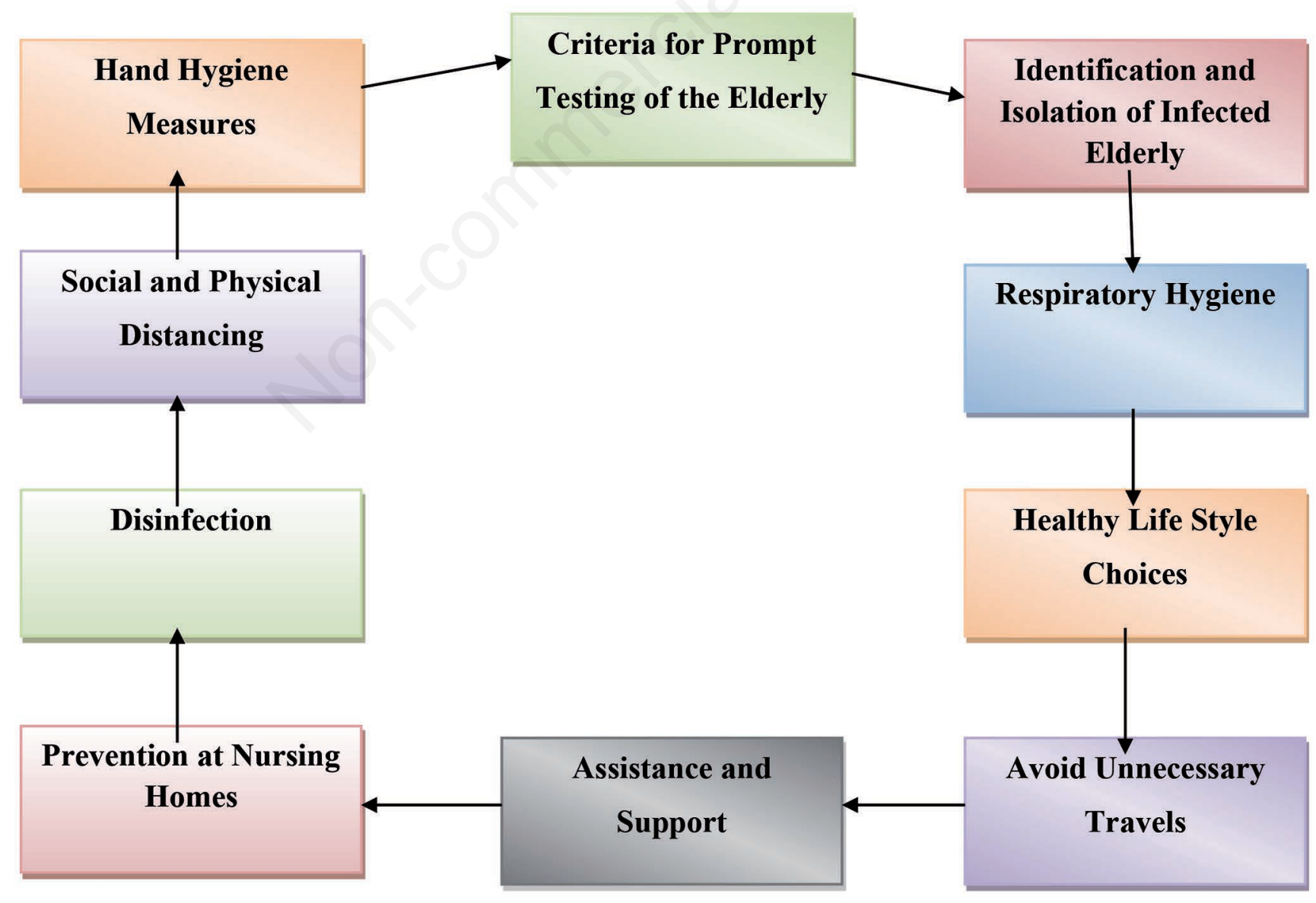

Figure 1. Methods for the prevention of COVID-19 for older adults. 
COVID-19 conditions. All geriatricians must be cautious and ought to consistently anticipate atypical symptoms of COVID-19 in patients of $>70$ years, and be aware that frail older adults with atypical symptoms have progressively adverse outcomes compared to older adults with typical symptoms. Self-isolation is the way toward avoiding public contact in order to diminish the spread of highly contagious infections. Self-isolation is advised for older adults with affirmed cases of COVID-19, and for individuals who have traveled to COVID19 hotspot territories in the last fourteen days. ${ }^{38}$ Furthermore, self-isolation is imperative for older adults who have come into contact with COVID-19 patients; individuals who are expecting their diagnosis and those that have had contact with individuals who have returned from COVID-19 hotspot territories. In the event that a nursing home occupant is confirmed to have COVID-19, with no obvious symptoms, they ought to be promptly moved to the assigned COVID-19 care unit. ${ }^{39}$ Occupants or roommates of residents with COVID-19 should anticipatorily be considered exposed and potentially infected, and should not cohabit with other residents except if they stay asymptomatic or have tested negative for SARS-CoV-2 in fourteen days after their last exposure. ${ }^{39}$ Furthermore, asymptomatic inhabitants and health care practitioners who were exposed to resident with COVID-19 ought to be considered infected and be isolated as a precautionary measure before diagnosis presents otherwise.$^{39}$ Also, self-quarantine is advised for older adults and individuals in COVID19 hot spots regions. ${ }^{40-41}$ Importantly, contact tracing must be performed to identify older adults who may have come into contact with confirmed COVID-19 patients, in other to decrease the spread of SARS-CoV2 and for prompt diagnosis of infection. ${ }^{6}$

\section{Social and physical distancing}

Social and physical distancing is the decrease or stoppage of physical contacts with individuals. During COVID-19 pandemic, Social and physical distancing is one of the significant ways for older adults to prevent contracting the disease. ${ }^{42}$ Social and physical distancing measures for older adults incorporates, decrease in the number of unnecessary visitors, staying indoors, having neighbors to get daily needs, foods and medicines. ${ }^{6}$ Significantly, social and physical distancing need not mean depression or social disconnection. Older adults ought not be disconnected during the COVID-19 pandemic, and social contact should in any case be kept up to prevent mental health problems. ${ }^{10}$ To prevent social seclusion and depression of older adults, children and relatives should call older adults frequently, older adults ought to take part in game activities, read books, watch television, listen to music and participate in minor physical exercises to the level of their health.

\section{Hand hygiene}

Hand hygiene is the thorough washing of hands to eliminate germs and microorganisms on the hands. To prevent COVID19 , older adults ought to consistently wash their hands with cleanser, soap and clean running water for twenty seconds before and after preparing and eating food, using the toilet, or touching any surfaces. Likewise, unwashed hands ought not to be used to contact any part of the face to prevent SARS-CoV-2 infection. ${ }^{10,42}$ Although hand washing is the better alternative, hand sanitizer may also be used to eliminate any germs and micro-organisms. Furthermore, older adults should bath daily, and ensure that cloths used are frequently changed, washed and dried appropriately before wearing them.

\section{Respiratory hygiene}

Respiratory hygiene is measures that ought to be taken by older adults to prevent the contagion of highly infectious respiratory infections. Such measures include coughing and sneezing in a tissue paper or a handkerchief, after which they ought to be appropriately discarded. ${ }^{6}$ In the event of inaccessibility of tissue paper or a handkerchief, older adults should cough and sneeze on bent elbow, and the hand ought to be completely washed with cleanser, soap and clean running water afterwards. ${ }^{6}$ Furthermore, older adults ought to avoid all high gathering places like markets, shopping centers, places of worships, etc. Significant distances ought to be maintained when talking, walking and sitting with people, and consistently get unwashed hands away from the face. . $^{10,42-44}$

\section{Disinfection of surfaces}

With the intensity of disease pathogens and microorganisms on surfaces, regular cleaning of surfaces is imperative to prevent the transmission of SARS-CoV-2. Older adults should routinely disinfect touchable surfaces at homes to eliminate germs. Additionally, handrails, sticks and walkers used at nursing homes by older adults ought to be normally cleaned to prevent SARSCoV-2 transmission, ${ }^{10,42}$ Chemicals, for example ethanol, isopropanol, sodium hypochlorite, hydrogen peroxide, and povidone-iodine are effective disinfectants to eliminate SARS-CoV-2. ${ }^{45}$

\section{Stoppage to unnecessary travels}

Until further notice, older adults ought to postpone all nonessential travel to prevent contracting COVID-19. Additionally, all insignificant visits to clinical staff workplaces ought to be decreased to prevent clinic transmission of SARS-CoV-2. Presently, the health care system is overwhelmingly compounded everywhere throughout the world as consequence of COVID-19 pandemic, hence, stoppage to unnecessary clinical visits ought to be guaranteed to avail medical workers the chance to concentrate more on extreme cases of COVID-19. For older adults with comorbidities and other geriatric conditions, adequate medications ought to be stored at home, but with strict caution or supervision against overdose.

\section{Wearing of face masks}

Protective face mask are materials worn around the face to cover the nose and mouth in other to prevent the transmission of contagious respiratory diseases. As prudent steps, the use of face cover is emphatically advised for individuals infected with COVID-19 to lessen the transmission of SARS-CoV-2 through sneezing, coughing, and talking. ${ }^{46}$ Also, wearing of protective face mask by uninfected older adults can help decrease the rate and odds of touching the face with infected and unwashed hands in COVID-19 hotspots zones, and will likewise diminish the odds of SARS-CoV-2 infection. ${ }^{46}$ Significantly, most health specialists in nations across continents are encouraging individuals to routinely wear face masks in public to forestall the spread of SARS-CoV-2.

\section{Healthy life style}

During this time of COVID-19 pandemic, older adults ought to engage in healthy life 
style choices to prevent contracting SARS$\mathrm{CoV}-2$ infection. Such measures include good and adequate nutrition to build and fortify the immune system to fight off infections, maintain regular physical activity to improve body fitness and to improve mental health, ensure regular sleeps, rest and avoid tobacco usage and alcohol consumption. ${ }^{8}$

\section{Assistance and support for older adults}

Older adults staying alone in the community needs food, medications, supports, etc., and this is the responsibilities of relatives, the government, NGOs, religious associations and other stakeholders but without compromising physical and social distancing. In all, it is imperative that individuals should remain connected with older adults during this period. Importantly, if keeping older adults safe implies no visitations, at that point they can be contacted through calls and different methods so they don't feel dismissed and forlorn. Social and physical distancing does not mean social segregation. Older adults need to have good comprehension of the continuous and routine COVID-19 preventive information been circulated in the media, and they should be kept abreast with the privilege and latest information, and participate in them to remain protected from COVID-19. ${ }^{8}$

\section{Older adults in nursing homes}

Nursing homes are residential places for individuals who require consistent nursing care, and have significant deficiencies with daily activities, particularly frailty older adults. Significantly, nursing homes are explicitly intended to take care of older adults in their entirety. Presently, it is not advisable to remove older adults from nursing home during this COVID-19 pandemic period, except if it is advised by health authorities, as placing older adults in different and new conditions may bring about the breakage of hip, and numerous different issues may emerge with moving them. Additionally, acclimatizing to the new condition may be time consuming, which may constantly affect their psychological, social and physical health. ${ }^{47,48}$ The breakout of SARS-CoV-2 infection has occurred in some nursing homes in the world, subsequently, there is a need for strict COVID-19 preventive measures in nursing homes across the globe. ${ }^{49}$ Statistically, in Spain $57 \%$ of all COVID-19 mortalities have occurred in nursing homes for older adults, $54 \%$ in Ireland, $45 \%$ in Italy, $45 \%$ in France, and $42 \%$ in Belgium, while there have been over $14 \%$ of COVID-19 confirmed cases among nursing home occupants in the United Kingdom. ${ }^{50}$ In the USA, over 3600 COVID-19 fatalities have infection outbreaks in nursing homes, which are currently a successive event, as over $40 \%$ of COVID-19 fatalities in Colorado State occurred among residents of nursing homes in the state..$^{51,52}$ Importantly, if there is a confirmed case of COVID-19 in a particular nursing home, all the inhabitants ought to be tested; thorough contact tracing ought to be guaranteed, thereafter, enormous and intensive disinfecting of the entire facility ought to be implemented to obliterate SARS-CoV$2 .{ }^{47}$ Furthermore, nursing home workers ought to be prepared by specialists on counteraction and control of infections, particularly SARS-CoV-2, and consistent protective gears and materials ought to be provided and used in nursing homes globally.

\section{Conclusions}

From this review study, it was uncovered that, firstly, as COVID-19 pandemic lingers on, older adults, particularly individuals with comorbidities and other geriatric conditions are confronting the severity of COVID-19 impacts with daily ceaseless increment in morbidity and mortality cases. Secondly, the severity of COVID-19 among older people is due to biological (reduced immunity with old age), socio-economic (neediness and overstretched health system) and physical reasons (frailty and comorbidities). Thirdly, there is a continuous increment in the numbers of SARS-CoV-2 transmission rates and COVID-19 fatalities in nursing homes globally; a place saddled with the responsibilities of older adults getting the best care and solace. Lastly, to mitigate the spread of COVID19 among older adults, strict adherence to social and physical distancing, frequent hand hygiene and respiratory hygiene, frequent disinfection of surfaces, stoppage to unnecessary travel and nonessential doctors visitations, wearing of face masks, healthy life style choices, proper identification and isolation of infected older adults, provision of assistance and support for older adults, and prevention of infections in nursing homes should all be implemented regularly globally.

\section{Recommendations}

Recommendations from all COVID-19 prescient models show that circumstance will improve. Being older adults the group that feels the overwhelming brunt of COVID-19 pandemic, the burden of protecting this vulnerable group of individuals in society falls on gerontologists, geriatricians, health educators, and other health workers, governmental and NGOs. In view of the result of this review, the accompanying suggestions are made: i) maximum compliance to COVID-19 preventive measures highlighted in this review ought to be ensured and implemented by older adults and be ensured by caregivers; ii) government, NGOs, family members and other relevant stakeholders ought to give supports to older adults during COVID-19 pandemic period; iii) geriatricians must be cautious and ought to consistently envisage atypical symptoms of COVID-19 in patients beyond 75 years of age globally; iv) studies ought to be done on inclining and reinforcing factors favoring high prevalence of COVID-19 pandemic in nursing homes, in order to get progressively suitable observational information that will enable informed preventive decisions, as nursing homes are the emerging channel of SARS-CoV-2 transmission and COVID-19 fatalities for older adults.

\section{References}

1. Lai C, Shih T, Ko W, et al. Severe acute respiratory syndrome coronavirus 2 (SARS- CoV-2) and coronavirus disease-2019 (COVID-19): the epidemic and the challenges. Int $\mathrm{J}$ Antimicrob Agents 2020;55:105924.

2. Lu R, Zhao X, Li J, et al. Genomic characterisation and epidemiology of 2019 novel coronavirus: implications for virus origins and receptor binding. Lancet 2020;395:565-74.

3. Wan Y, Shang J, Graham R, et al. Receptor recognition by the novel coronavirus from wuhan: an analysis based on decade-long structural studies of SARS coronavirus. J Virol 2020;94.

4. Zhang T, Wu Q, Zhang Z. Probable pangolin origin of SARS-CoV-2 associated with the COVID-19 outbreak. Curr Biol 2020;30:1346-51.e2.

5. Auwaerter PG. Coronavirus COVID-19 (SARS-CoV-2). 2020. [Accessed 2020 June 25]. Available from: https://www. hopkinsguides.com/hopkins/view/Johns _Hopkins_ABX_Guide/540747/all/Coro navirus_COVID_19_SARS_CoV_2_

6. Ayenigbara IO. COVID-19: an international public health concern. Cent Asian J Glob Health 2020;9 [Epub ahead of print].

7. Cook TM. Personal protective equipment during the COVID-19 Pandemic - 
A Narrative Review. Anaesthesia 2020 [Epub ahead of print].

8. World Health Organization. Statement Older people are at highest risk from COVID-19, but all must act to prevent community spread; 2020. Available from: http://www.euro.who.int/en/health -topics/health-emergencies/coronaviruscovid-19/statements/statement-olderpeople-are-at-highest-risk-from-covid19,-but-all-must-act-to-prevent-community-spread Accessed: 2020 June 25.

9. Chen TL, Dai Z, Mo P, et al. Clinical characteristics and outcomes of older patients with coronavirus disease 2019 (COVID-19) in Wuhan, China (2019): a single-centered, retrospective study. Gerontol A Biol Sci Med Sci 2020 [Epub ahead of print].

10. Arbaje A. Coronavirus and COVID-19: care giving for the elderly; 2020. Available from: https://www.hopkinsmedicine.org/health/conditions-anddiseases/coronavirus/coronavirus-caregiving-for-the-elderly Accessed: 2020 June 25.

11. Hussain A, Bhowmik B, Cristina do Vale Moreira N. COVID-19 and diabetes: knowledge in progress. Diabetes Res Clin Pract 2020;108142.

12. Zhang Y. The epidemiological characteristics of an outbreak of 2019 novel coronavirus diseases (COVID-19) - China, 2020; 2020. Available from: http://weekly.chinacdc.cn/en/article/id/e53946e2c 6 c 4- 41 e 9-9a 9b-fea 8db 1 a 8 f 51 Accessed: 2020 June 25.

13. Verity R, Okell LC, Dorigatti I, et al. Estimates of the severity of coronavirus disease 2019: a model-based analysis. Lancet Infect Dis 2020;20:669-77.

14. Center for Disease Control and Prevention. Severe outcomes among patients with coronavirus disease 2019 (COVID-19) - United States, February 12-March 16, 2020. Available from: https://www.cdc.gov/mmwr/volumes/69 /wr/mm6912e2.htm\#T1_down Accessed: 2020 June 25.

15. Jeske SS, Schuler PJ, Doescher J, et al. 2020. Age-related changes in T lymphocytes of patients with head and neck squamous cell carcinoma. Immun Ageing 2020;17.

16. David P, Drabczyk-Pluta M, Pastille E. Combination immunotherapy with antiPD-L1 antibody and depletion of regulatory $\mathrm{T}$ Cells during acute viral infections results in improved virus control but lethal immunopathology. PLoS Pathog 2020;16:e1008340.

17. Begley S. What explains Covid-19's lethality for the elderly? Available from: https://www.statnews.com/2020/03/30/ what-explains-coronavirus-lethalityfor-elderly/ Accessed: 2020 June 25.

18. Loh L, Gherardin NA, Sant S, et al. 2020. Human mucosal-associated invariant $\mathrm{T}$ cells in older individuals display expanded TCR $\alpha \beta$ clonotypes with potent antimicrobial responses. J Immunol 2020;204:1119-33.

19. Sawalha AH, Zhao M, Coit P, et al. Epigenetic dysregulation of ACE2 and interferon-regulated genes might suggest increased COVID-19 susceptibility and severity in lupus patients. Clin Immunol 2020;215:108410.

20. Bizzarri M, Laganà AS, Aragona D, et al. Inositol and pulmonary function. Could myo-inositol treatment downregulate inflammation and cytokine release syndrome in SARS-CoV-2?. Eur Rev Med Pharmacol Sci 2020;24:3426-32.

21. Hadadi A, Mortezazadeh M, Kolahdouzan K, et al. Does recombinant human erythropoietin administration in critically ill COVID-19 patients have miraculous therapeutic effects? J Med Virol 2020;92:915-8.

22. Márquez EJ, Chung $\mathrm{C}$, Marches R. et al. Sexual-dimorphism in human immune system aging. Nat Commun 2020;11:751.

23. World Economic Forum. An expert explains: how to help older people through the COVID-19 pandemic; 2020. Available from: https://www. weforum.org/agenda/2020/03/coronavirus-covid-19-elderly-older-peoplehealth-risk/ Accessed: 2020 June 25.

24. Nania R. Coronavirus and older adults: your questions answered; 2020. Available from: https://www.aarp.org/health/ conditions-treatments/info-2020/cdccovid-19.html Accessed: 2020 June 25.

25. Chung CJ, Nazif TM, Wolbinski M. The restructuring of structural heart disease practice during the Covid-19 pandemic. J Am Coll Cardiol 2020:75:2974-83.

26. Barrasa H, Rello J, Tejada S. SARSCov-2 in Spanish intensive care: early experience with 15-day survival in Vitoria. Anaesth Crit Care Pain Med 2020;S2352-5568(20)30064-3.

27. Malavolta M, Giacconi R, Brunetti D, et al. Exploring the relevance of senotherapeutics for the current SARSCoV-2 emergency and similar future global health threats. Cells 2020;9:909.

28. Feng Y, Ling Y, Bai T, et al. COVID-19 with different severity: a multi-center study of clinical features. Am J Respir Crit Care Med 2020;201:2380-8.

29. Lippi G, Mattiuzzi C, Sanchis-Gomar F, et al. Clinical and demographic characteristics of patients dying from COVID19 in Italy versus China. J Med Virol 2020 [Epub ahead of print].
30. Center for Disease Control and Prevention. COVID-NET: COVID-19Associated Hospitalization Surveillance Network, Centers for Disease Control and Prevention; 2020. Available from: https:/gis.cdc.gov/grasp/COVIDNet/CO VID19 3.html Accessed: 2020 June 25.

31. New York State Department of Health. NYS-COVID-19 tracker. 2020. Available from: https://covid19tracker.health.ny. gov/views/NYS-COVID19-Tracker/ NYSDOHCOVID-19Tracker-Fatalities? $\% 3$ Aembed $=$ yes $\& \% 3$ Atoolbar $=$ no $\& \% 3$ Atabs $=n$ Accessed: 2020 June 25.

32. John Hopkins University. Coronavirus COVID-19 Global Cases by the Center for Systems Science and Engineering (CSSE) at Johns Hopkins University (JHU); 2020. Available from: https:// gisanddata.maps.arcgis.com/apps/opsdashboard/index.html\#/bda7594740fd4 0299423467b48e9ecf6 Accessed: 2020 June 25.

33. Lloyd-Sherlock PG, Kalache A, McKee $\mathrm{M}$, et al. WHO must prioritise the needs of older people in its response to the Covid-19 pandemic. BMJ 2020;368 (m1164).

34. World Health Organization. Coronavirus disease (COVID-19) advice for the public; 2020. Available from: https://www. who.int/emergencies/diseases/novelcoronavirus-2019/advice-for-public Accessed: 2020 June 25.

35. Nguyen S, Major K, Cochet C, et al. [COVID-19 Infection in the Elderly in French-speaking Switzerland: An Inventory of Beliefs, Convictions and Certainties]. Rev Med Suisse 2020;16: 835-8.

36. Rikkert M, Vingerhoets RW, Geldorp $\mathrm{NV}$, et al. [Atypical clinical picture of COVID-19 in older patients]. Ned Tijdschr Geneeskd 2020;164:D5004.

37. Dadamo H, Yoshikawa T, Ouslander JG. Coronavirus disease 2019 in geriatrics and long-term care: the ABCDs of COVID-19. J Am Geriatr Soc 2020;68: 912-7.

38. Sjödin H, Wilder-Smith A, Osman S, et al. Only strict quarantine measures can curb the coronavirus disease (COVID19) outbreak in Italy, 2020. Euro Surveill 2020;25:2000280.

39. Center for Disease Control and Prevention. Coronavirus Disease 2019. Responding to Coronavirus (COVID-19) in Nursing Homes; 2020. Available from: https://www.cdc.gov/coronavirus/ 2019-ncov/hcp/nursing-homes-responding.html Accessed: 2020 June 25.

40. Anderson RM, Heesterbeek H, Klinkenberg D, et al. How will countrybased mitigation measures influence the 
course of the COVID-19 epidemic? Lancet 2020;395:931-4.

41. Qualls N, Levitt A, Kanade N, et al. Community mitigation guidelines to prevent pandemic influenza - United States, 2017. MMWR Recommend Rep 2017;66:1-34.

42. Davenne E, Giot JB, Huynen P. Coronavirus and COVID-19: focus on a galopping pandemic. Rev Med Liege 2020;75:218-25.

43. Malhotra N, Gupta N, Ish S, et al. COVID-19 in intensive care. Some necessary steps for health care workers. Monaldi Arch Chest Dis 2020;90 [Epub ahead of print].

44. Dexter F, Parra MC, Brown JR, et al. 2020. Preoperative COVID-19 defense: an evidence-based approach for optimization of infection control and operating room management. Anesth Analg 2020 [Epub ahead of print].

45. Kampf G, Todt D, Pfaender S, et al. Persistence of coronaviruses on inani- mate surfaces and their inactivation with biocidal agents. J Hospital Infect 2020;104:246-51.

46. World Health Organization. Coronavirus disease (COVID-19) advice for the public: when and how to use masks; 2020. Available from: https://www.who.int/ emergencies/diseases/novel-coronavirus-2019/advice-for-public/whenand-how-to-use-masks Accessed: 2020 June 25.

47. O'Neill D. Protecting our longevity dividend during Covid-19. Ir Med J 2020;113:50.

48. Kunz R, Minder M. COVID-19 pandemic: palliative care for elderly and frail patients at home and in residential and nursing homes. Swiss Med Wkly 2020;150:w20235.

49. Center for Disease Control and Prevention. Coronavirus disease 2019: nursing homes \& long-term care facilities; 2020. Available from: https:// www.cdc.gov/coronavirus/2019-ncov/ hcp/long-term-care.html Accessed: 2020 June 25.

50. The Guardian. Half of coronavirus deaths happen in care homes, data from EU suggests; 2020. Available from: https://www.theguardian.com/world/20 20/apr/13/half-of-coronavirus-deathshappen-in-care-homes-data-from-eusuggests Accessed: 2020 June 25.

51. Daily Mail. More than 3,600 elderly people have died from coronavirus in nursing homes across the US; 2020. Available from: https://www.dailymail.co.uk/news/article-8213307/More3-600-deaths-linked-coronavirus-outbreaks-nursing-homes.html Accessed: 2020 June 25.

52. Tabachnik S. Nearly $40 \%$ of coronavirus deaths in Colorado linked to nursing homes, long-term care facilities; 2020. Available from: https://www. denverpost.com/2020/04/11/coronavirus-covidcolorado-nursing-homes-deaths/ Accessed: 2020 June 25. 